\title{
Development of Microfluidic Systems for Fabricating Cellular Multilayers
}

\author{
Koji Matsuura, ${ }^{* \dagger}$ Ikuyo Sugimoto, ${ }^{*}$ Yuka Kuroda, ${ }^{*}$ Koji KadowaKI, ${ }^{* *}$ Michiya MatsusaKi,** \\ and Mitsuru AKASHI**
}

*Research Core for Interdisciplinary Sciences, Okayama University, 3-1-1 Tsushima-Naka, Kita, Okayama 700-8530, Japan

**Graduate School of Engineering, Osaka University, 2-1 Yamadaoka, Suita, Osaka 565-0871, Japan

\begin{abstract}
We designed a microfluidic system comprising microfluidic channels, pumps, and valves to enable the fabrication of cellular multilayers in order to reduce labor inputs for coating extracellular matrices onto adhesive cells (e.g., centrifugation). Our system was used to fabricate nanometer-sized, layer-by-layer films of the extracellular matrices on a monolayer of C2C12 myoblasts. The use of this microfluidic system allowed the fabrication of cellular multilayers in designed microfluidic channels and on commercial culture dishes. The thickness of the fabricated multilayer using this microfluidic system was higher than that of the multilayer that was formed by centrifugation. Because cellular multilayer fabrication is less laborious and the mechanical force to the cell is reduced, this novel system can be applied to tissue modeling for cell biology studies, pharmaceutical assays, and quantitative analyses of mechanical or chemical stimuli applied to multilayers.
\end{abstract}

Keywords Layer-by-layer, extracellular matrix, cellular multilayer, microfluidic system

(Received June 3, 2016; Accepted July 12, 2016; Published November 10, 2016)

\section{Introduction}

In tissue engineering, mimicking the cellular environment is important for restoring the functions of freshly isolated cells and regenerating tissue-engineered cell constructs. ${ }^{1}$ Most cells exist as multilayers under physiological conditions. The deposition of layer-by-layer (LbL) films of the extracellular matrix (ECM) over cultured cells is an important step in preparing cellular multilayers. ${ }^{2-4}$ Microfluidic devices and systems have been developed to fabricate three-dimensional (3D) cellular structures, including cellular multilayers, and to assess how cells sense changes in their microenvironment. ${ }^{5-11}$ Owing to the availability of soft lithography, a convenient method for microscale fabrication of microfluidic channels has been developed for advancing such research. Microfluidic cell culture systems designed to study cellular responses to external stimuli have been used to investigate the effects of ECM, chemotaxis, and force transduction. ${ }^{6-11}$ Microfluidic 3D cellular structure fabrication and culture systems are potentially advantageous research tools for developing rapid and reproducible assays of small-volume samples without the need for performing laborintensive procedures. ${ }^{11}$

Matsusaki et al. reported on cell coating with ECMs by centrifugation to fabricate a cellular multilayer following a

† To whom correspondence should be addressed.

E-mail: kmatsuura@bme.ous.ac.jp

Present Address: Department of Biomedical Engineering, Faculty of Engineering, Okayama University of Science, 1-1 Ridai-Cho Kita, Okayama 700-0005, Japan. high-throughput method (Fig. 1A). ${ }^{12-14}$ The advantage of using the LbL centrifugation method is its easy handling. This technique can be easily performed by a person who is a novice in cell manipulation. However, the centrifugation process for $\mathrm{LbL}$ is laborious because it requires at least seven centrifugation steps, and it potentially induces negative effects by causing some cells to grow along the hands of the centrifugal force and form cellular pellets. ${ }^{14}$ Thus, we developed an LbL-coating process using a programmed microfluidic system. This process can reduce the volume of ECM solutions and is less laborious than the centrifugation process.

In this study, we developed microfluidic systems comprising microfluidic channels and electrical devices for fabricating an LbL film of ECM and a cellular bilayer. Figure 1B illustrates the functioning of this system, which can be used for the development of cellular multilayers in a microfluidic channel and on a culture dish. The system comprises electric devices (Fig. 1C) and microfluidic channels on glass or glass-bottomed dishes (Fig. 1D). LbL ECM nanofilms were directly prepared on the cell surface, on which they acted as a stable adhesive for deposition of the second cell layer. ${ }^{14}$ We fabricated cellular bilayers in a microfluidic channel using the proposed system. We also developed an LbL cell-coating process in a channel, and the coated cells were seeded into a culture dish. These technologies can be applied to analyses such as tissues for modeling in cell biology, pharmaceutical assays, and cellular responses to chemical and/or mechanical stimuli. 


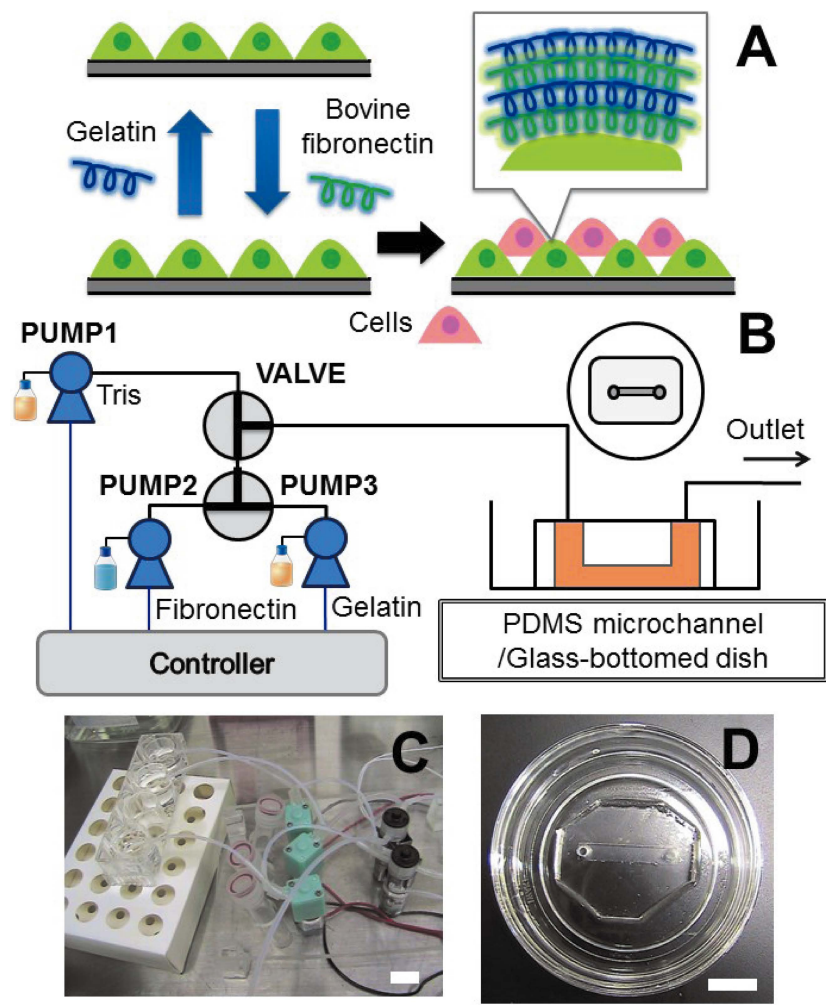

Fig. 1 (A) Schematic representation of the methods for preparing cellular multilayers. (B) Schematic displaying the microfluidic system. The black and blue lines show the fluidic channels and electrical lines, respectively. (C) Pumps and valves used to fabricate a layer-by-layer film in a microfluidic channel. (D) A poly(dimethylsiloxane) microfluidic channel on a glassbottomed dish. The bars indicate $1 \mathrm{~cm}$.

\section{Experimental}

Microfluidic channel design, and protocols for cell culture in this channel and on a culture dish

Figures 2A and 2B show the dimensions and a photograph of the microfluidic channel, respectively. The microfluidic channel for the layered cell culture was a poly(dimethylsiloxane) (PDMS)-based channel combined with a cover glass at the bottom. The length, height, and width of the channel were 1.5, 0.2 , and $0.8 \mathrm{~mm}$, respectively (Fig. 2A). Figure $2 \mathrm{C}$ shows the coating and culture methods for fabricating cellular multi-layers in the channel. To prevent the medium in the microfluidic channel from drying, the cells in the microfluidic channel were cultured by soaking the channel in a $35-\mathrm{mm}$ dish containing the medium.

Figures 2D and 2E show the dimensions and a photograph of the microfluidic channel for cell coating with ECM, respectively. We designed the PDMS-based microfluidic channel for the LbL method, and formed an inlet center region parallel to the flow direction from the inlet to the outlet. Because the density of mammalian cells $\left(1.05-1.08 \mathrm{~g} / \mathrm{cm}^{3}\right)$ is higher than that of the medium, these round cells were settled by gravitational and/or fluid mechanical forces and then attached to the bottom of the microfluidic channel. ${ }^{15,16}$ The volume of the channel region where the cells were coated was approximately $1 \mathrm{~mL}$. A larger volume for this region is more preferable for coating a higher number of cells. However, when the volume increases, localized turbulence tends to occur. At a flow rate of $0.35 \mathrm{~mL} / \mathrm{min}$, the cells did not move under laminar fluid flow. If the channel flow is not laminar with turbulence, round cells at the bottom of the channel would be lifted up by turbulence and leave the microfluidic channel. As flow rate increases, damage to the
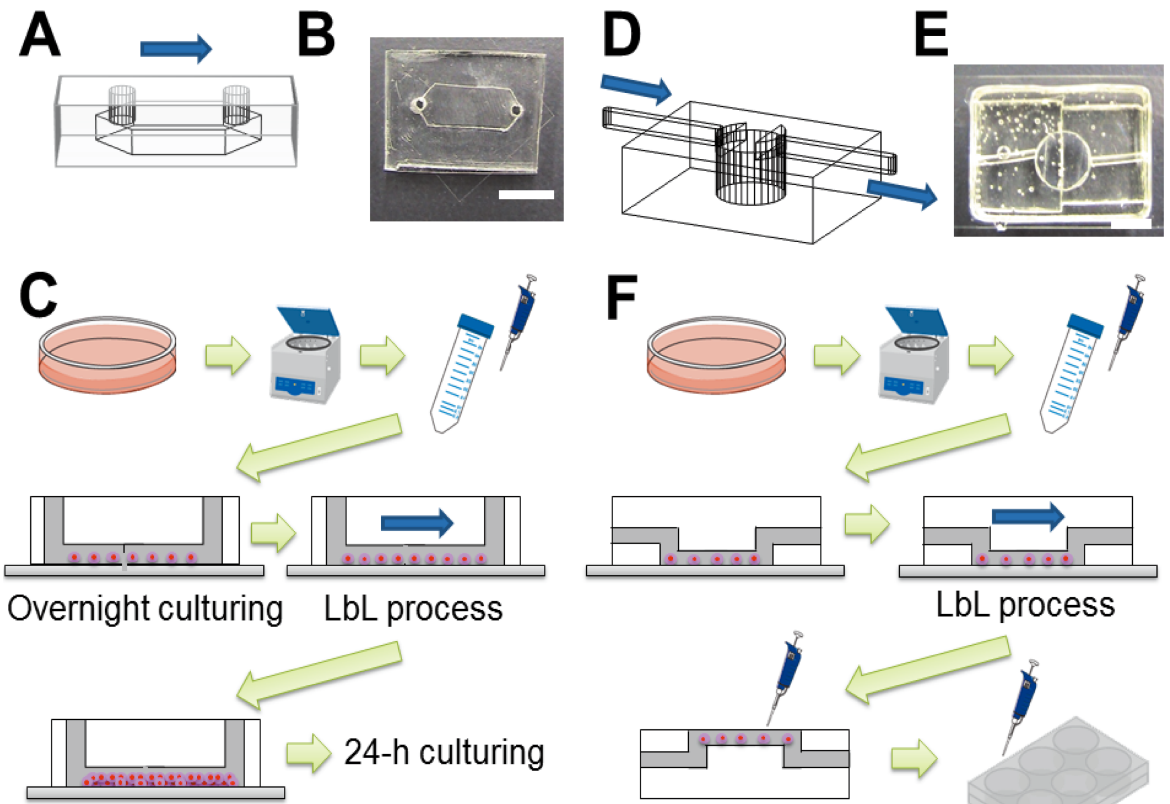

Add cells on the first layer

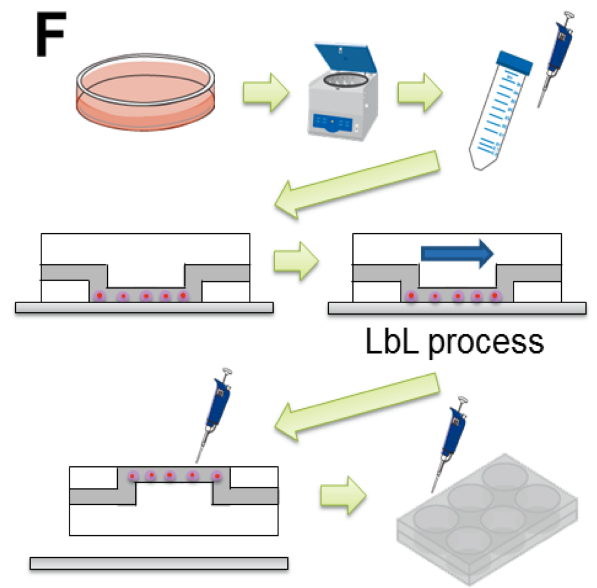

Fig. 2 (A) Cellular bilayer preparation in a microfluidic channel: structure of the channel. (B) Photograph of the channel (A). (C) Schematic representation of the experimental protocol for the preparation of a cellular bilayer in a microfluidic channel. (D) Structure of the microfluidic channel used for preparing the layer-by-layer (LbL) extracellular matrix (ECM) film using the microfluidic system. (E) Photograph of the channel (E). (F) Schematic representation of the experimental protocol for the preparation of an LbL ECM film using the microfluidic system. 
cells also increases, induced by shear stress. We would like to take at least $1 \mathrm{~min}$ to coat ECMs in one process. The recommended maximum flow rate is [microfluidic channel volume around cells $(\mathrm{mL})] /(1 \mathrm{~min})$, which is usually $1 \mathrm{~mL} / \mathrm{min}$. Therefore, cells at the bottom could be coated with ECMs by the LbL method. Figure $2 \mathrm{~F}$ shows the protocol for the cell coating method using this system and cell culture of the coated cells.

\section{Microfluidic channel preparation}

The microfluidic channels were prepared from PDMS (TSE 3032; Momentive Performance Materials, Tokyo, Japan). After degassing PDMS under vacuum for at least $30 \mathrm{~min}$, it was cured in an oven at $70^{\circ} \mathrm{C}$ for $60 \mathrm{~min} \cdot{ }^{17-19}$ The PDMS microchannel substrate was cured as described above, and access holes $(2 \mathrm{~mm}$ in diameter) were created through the elastomer to enable the fluid to be applied to the microchannels. The PDMS substrate was subsequently bonded to a glass slide after plasma treatment for $30 \mathrm{~s}$ (PDC-32G; Harrick Plasma Inc., Ithaca, NY). ${ }^{18}$

\section{Cell culturing conditions before the LbL processes}

$\mathrm{C} 2 \mathrm{C} 12$ myoblasts were maintained in a culture as previously described. ${ }^{20}$ Briefly, the cells were grown in Dulbecco's modified Eagle's medium (DMEM; Sigma, St. Louis, MO) containing 10\% fetal bovine serum (FBS; GIBCO, Eggenstein, Germany). The microfluidic channel was incubated with $0.2 \mathrm{mg} / \mathrm{mL}$ bovine fibronectin (BFN; CHEMICON, Temecula, $\mathrm{CA})$ for $30 \mathrm{~min}$. The cells were harvested by centrifugation, and $10-20 \mu \mathrm{L}$ of the resuspended cell pellet was injected into the microfluidic channel. We prepared the cell suspension as a confluent condition and applied the suspension with $10^{5}-10^{6}$ cells $/ \mathrm{mL}$ to the microfluidic channel. The cells in the microfluidic channel were incubated overnight at $37^{\circ} \mathrm{C}$.

Preparation of the LbL ECM film using the microfluidic system and fabrication of layered structures in microfluidic channels

The microfluidic system used to deposit the LbL ECM comprised a microfluidic channel with an inlet and outlet. The system consisted of three micropumps, two solenoid valves (Fig. 1C), and a custom-made controller. BFN, gelatin (GEL), and washing buffer were added to the reservoirs. The flow of the washing buffer into the microfluidic channel was regulated using an electric controller equipped with a one-chip microcomputer. Solutions of 50-mM Tris- $\mathrm{HCl}$ ( $\mathrm{pH} 7.4)$, $0.05-\mathrm{mg} / \mathrm{mL}$ BFN, and 0.2-mg/mL GEL (Nacalai Tesque, Inc., Kyoto, Japan) were added to the chambers connected to the micropump. We prepared a delivery program and loaded BFN and GEL solutions as well as the washing buffer into the microfluidic channel to generate an LbL artificial ECM film on the cellular layer. We separately coated the cells using the ECM solutions. At first, we used BFN with the strong adhesion characteristics to substrates. A flow chart of the solution handling process is shown in Fig. 3.

The inlet and outlet of the microfluidic channel containing the attached cells were connected with poly(tetrafluoroethylene) (PTFE) tubing to a solenoid valve, micropump, and reservoirs containing the ECM solution and washing buffer. The PTFE tubing and reservoirs were sterilized with ethanol and dried prior to use. The culture medium in the microfluidic channel was removed using micropipettes, and the program for the LbL process was then initiated. The LbL process was repeated four times, following which $0.35 \mathrm{~mL}$ of $50-\mathrm{mM}$ Tris- $\mathrm{HCl}(\mathrm{pH} 7.4)$ was loaded into the microfluidic channel. After stopping the electric controller, the channel was disconnected from the PTFE tubes and the solution in the channel was removed with a

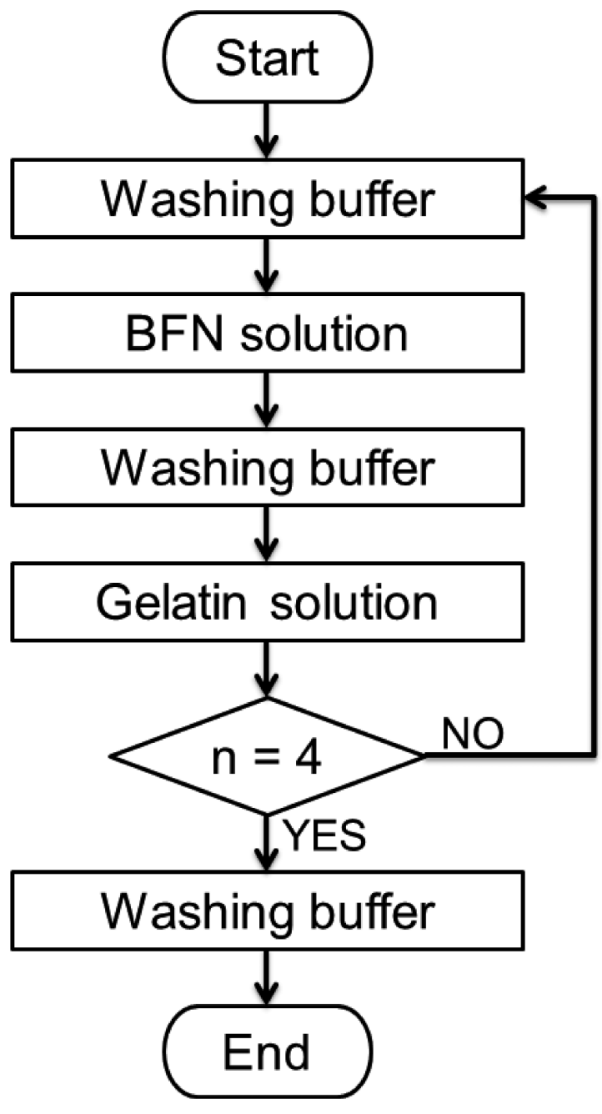

Fig. 3 Flow chart of the LbL process using the electric controller.

micropipette. These experimental processes are summarized as shown in Fig. 2C.

After preparing the LbL ECM film, layered structures of mouse myoblasts ( $\mathrm{C} 2 \mathrm{C} 12$ cells) were fabricated in the microfluidic channel with a micropipette. The cells were fixed with paraformaldehyde, stained with fluorescent dyes, and observed under a multicolor confocal laser fluorescent microscope (CLFM; FluoView 1000, Olympus Co., Ltd., Tokyo, Japan).

Preparation of coated C2C12 myoblasts with an LbL ECM film in the PDMS microfluidic channel by the centrifugation method

The microfluidic system was used to coat the $\mathrm{C} 2 \mathrm{C} 12$ cells with an LbL ECM film in the PDMS microfluidic channel (Fig. 2F). The $\mathrm{C} 2 \mathrm{C} 12$ cells were injected into the PDMS microfluidic channel and, after $10-15 \mathrm{~min}$, they settled at the bottom. Then, the program for the microfluidic system was started. BFN or GEL of a volume equivalent to that of the PDMS microfluidic channel was added alternately, followed by washing with $50-\mathrm{mM}$ Tris- $\mathrm{HCl}$ of a volume two times that of the PDMS microfluidic channel. This process was repeated four times as shown in Fig. 3. After stopping the electric controller and removing the PTFE tubes, the solution in the PDMS microfluidic channel was removed with a micropipette. The PDMS slab with the microfluidic channel was removed from the glass slide and the channel was inverted to resuspend and recover the cells in DMEM with a micropipette. Cells were centrifuged at $100 \mathrm{~g}$. Before coating, the $\mathrm{C} 2 \mathrm{C} 12$ cells were stained with CellTracker Red. Coated C2C12 cells at different concentrations were cultured on DMEM containing 10\% FBS in a 35-mm dish or a 96-well microtiter plate in the presence of $5 \% \mathrm{CO}_{2}$ at $37^{\circ} \mathrm{C}$ for $24 \mathrm{~h}$. 


\section{Confocal laser fluorescent microscope (CLFM)}

The $\mathrm{C} 2 \mathrm{C} 12$ cellular multilayer were stained with a phalloidinAlexaFluoro 488 conjugate (Pd-AF488), CellTracker Blue, and/or Rhodamine 2 (Rhod-2; Invitrogen, Carlsbad, CA). Pd-AF488 stained the cytoskeletons of the fixed cells and Rhod-2 stained all the cells in the multilayer. Also, 4',6-diamidino-2-phenylindole (DAPI) was used for nuclear staining. To evaluate the cells across the depths of the microfluidic channel and within the cell layers, cross-sectional fluorescent images were obtained.

\section{Analysis of cell heights from z-stacked fluorescence images}

The height of the cytosol in the observed cells was estimated based on the z-stacked confocal fluorescent images in the coating experiment that used the microfluidic channel. The average CellTracker Red fluorescence intensity in each frame was analyzed using the Image $\mathbf{J}$ software (National Institutes of Health, Bethesda, MD). All cells in the multilayer were stained with CellTracker Red. The relationship between the height of the focus for confocal scanning (horizontal axis) and the average fluorescence intensity (vertical axis) was investigated. The data points were fitted by a Gaussian function as shown in Eq. (1).

$$
I(h)=a \exp \left\{\frac{-(h-b)^{2}}{2 w^{2}}\right\}+c
$$

The widths of the bell $w, a, b$, and $c$ are the fitting parameters. $I(h)$ and $h$ denote the average fluorescence intensity and the height of the confocal plane from the bottom, respectively. We considered the height of the layered cells as being the full width at half maximum (FWHM). The FWHM was calculated according to Eq. (2).

$$
\mathrm{FWHM}=2 \sqrt{2 \ln 2} w=2.35 w
$$

In this study, FWHM was considered as the height of the 3D cellular structure. We compared FWHMs of 3D cellular structures fabricated using the centrifugation and the microfluidic coating methods.

\section{Statistical analyses}

The intercepts of the linear regression analyses between the data from the microfluidic system and the centrifugation method were compared by employing one-way analysis of covariance (ANCOVA). ${ }^{21,22}$ A $P$-value of $<0.05$ was considered statistically significant.

\section{Results and Discussion}

A multilayer of the $\mathrm{C} 2 \mathrm{C} 12$ myoblasts was fabricated in a microfluidic channel. One hour after the injection, we checked the elongation of $80-90 \%$ of the $\mathrm{C} 2 \mathrm{C} 12$ cells on the microfluidic channel using microscopic observation. CLFM images of a stained confluent cellular multilayer without fixation are shown in Figs. 4A and 4B. CellTracker Blue stained the cells in the lower layer, whereas Rhod-2 stained all the cells. Figures 4C and 4D show the lower and upper layers, respectively, of the $\mathrm{C} 2 \mathrm{C} 12$ cellular bilayer. The height of the bilayers was approximately $10-20 \mu \mathrm{m}$. Using this system, the cellular bilayer with migrated cells could be prepared in the microfluidic channel.

We compared the thickness of the cellular multilayer by the microfluidic ECM-coating method and the conventional centrifugation method (Fig. 5). The average fluorescence

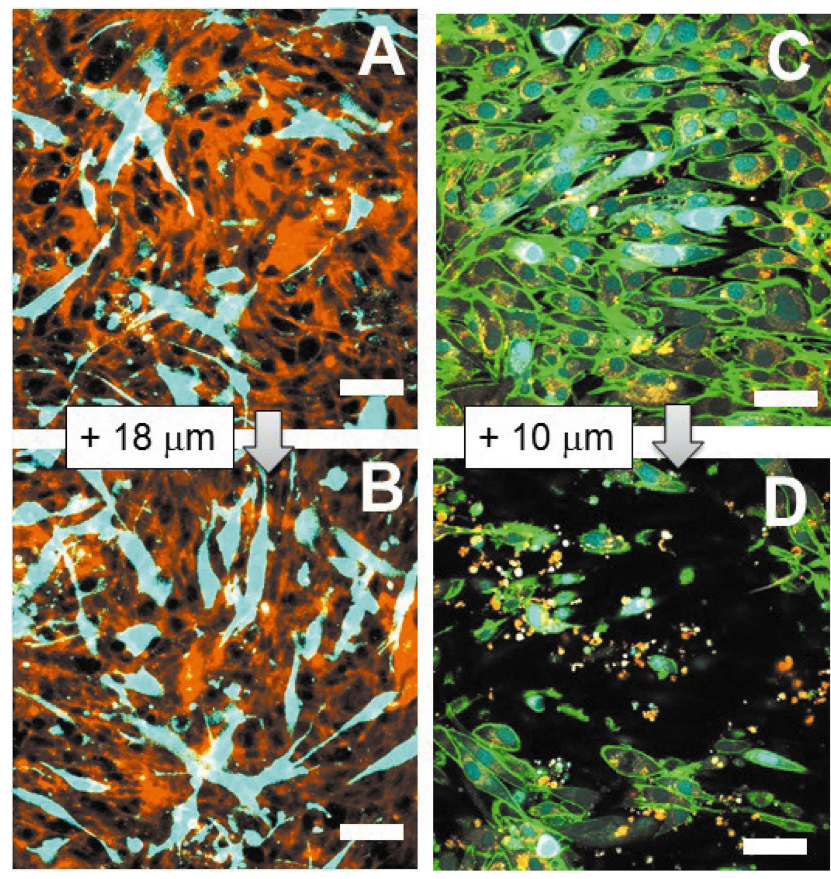

Fig. 4 Multicolor confocal laser fluorescent microscope images of fixed C2C12 cells. (A) and (B) Light blue and brown fluorescence emissions of cells stained with CellTracker Blue and Rhod-2, respectively. The distance of depths of focus between these images was $18 \mu \mathrm{m}$. (C) and (D) Green and light blue fluorescence emissions of cells stained with Pd-AF488 and 4',6-diamidino-2-phenylindole (DAPI), respectively. The distance of depths of focus between these images was $10 \mu \mathrm{m}$.

intensity was fitted by a Gaussian function expressed in Eq. (2), and FWHM was approximated to the thickness of the cellular multilayer. Correlations were observed between the thickness of the cellular multilayer and the seeded cell concentration in both groups: $R^{2}=0.51$ and 0.46 for the microfluidic system and the centrifugation method, respectively. For the regression coefficient of the intercept, which was indicative of the thickness of the cellular multilayer, a significant difference between the two groups was observed $(P<0.05)$. The regression coefficients of the microfluidic system and centrifugation groups were 14 and $12 \mu \mathrm{m}$, respectively. Furthermore, the slopes of these linear regression analyses were 0.0024 and $0.0021\left(\mu \mathrm{m} \times \mathrm{mm}^{2} /\right.$ cells $)$ for the microfluidic system and centrifugation groups, respectively. We concluded that the $3 \mathrm{D}$ structures prepared by this microfluidic method were slightly higher than those obtained by the centrifugation method.

This system can be used to reduce the mechanical stimuli to the cells by controlling the microfluidic channel dimension and flow rate. In addition, the characteristics of this system could be beneficial for manipulating cells sensitive to mechanical stimuli. The calculated fluid shear stress (FSS) of the cells at the bottom of the microfluidic channel was approximately $0.02 \mathrm{dyn} / \mathrm{cm}^{2}$ according to the following Eq. (3): ${ }^{23}$

$$
\mathrm{FSS}=\frac{6 \mu Q}{b a^{2}}
$$

where $Q$ is the flow rate; $\mu$ is the viscosity of water $(0.8 \mathrm{mPa} / \mathrm{s}$ at $30^{\circ} \mathrm{C}$ ); and $a$ and $b$ are the height and width of the microfluidic channel, respectively. In this flow experiment for the LbL process in the microfluidic channel, the values of $Q, a$, and $b$ 

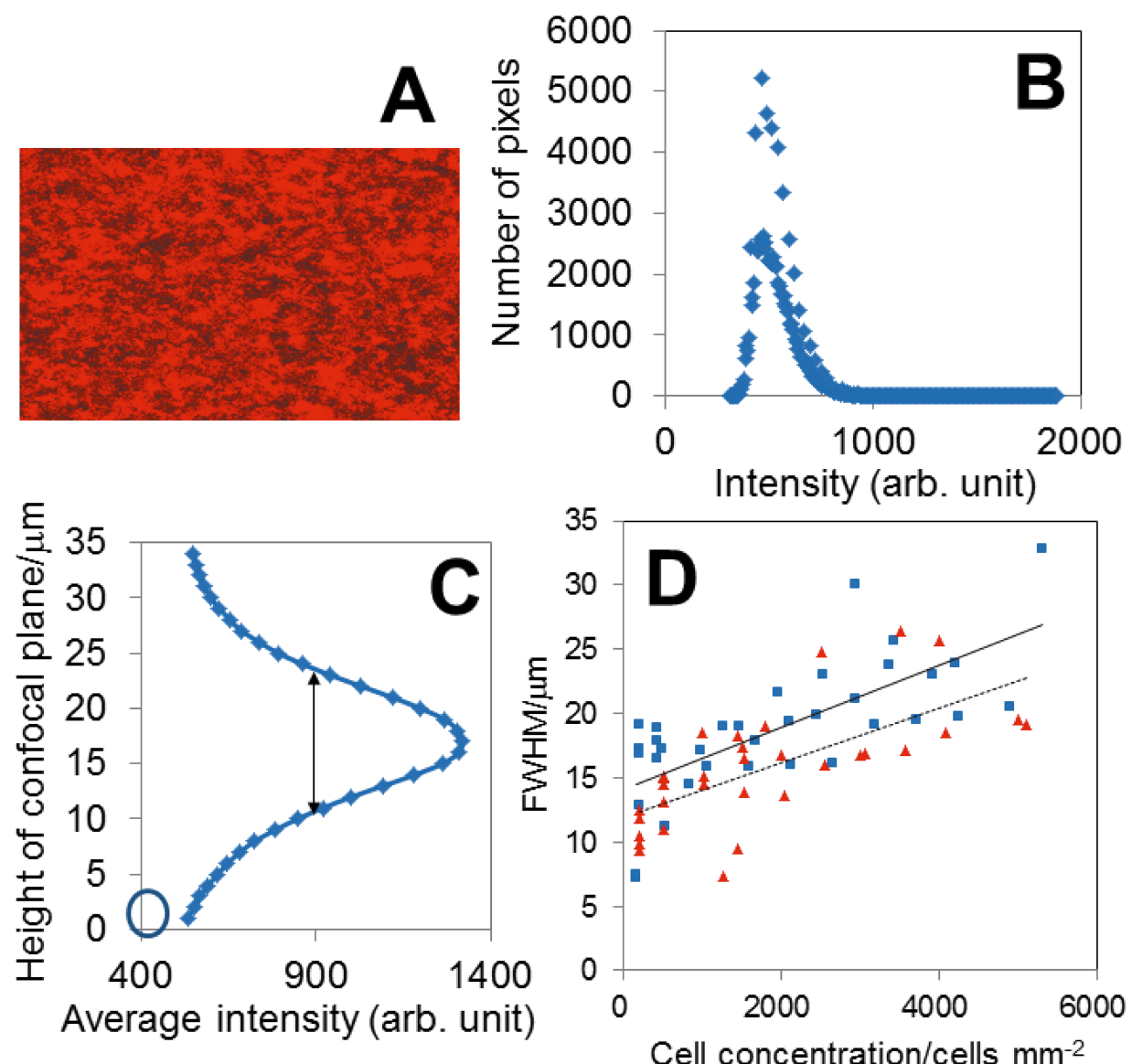

Fig. 5 (A) Representative image of $\mathrm{C} 2 \mathrm{C} 12$ cellular structures stained with CellTracker Red. (B) Histogram of the fluorescence intensities in the image. (C) Relationship between average intensity of fluorescence and height of the confocal plane. The horizontal and vertical axes represent the average fluorescence intensities in the z-stacked image and the height of focus from the bottom, respectively. The graphs were fitted using a Gaussian function, and the full width at half maximum (FWHM) was regarded as being the height of the 3D cellular structure. (D) Comparison of FWHMs of 3D cellular structures obtained using the microfluidic system and the centrifugation method. The horizontal and vertical axes represent the cell concentrations for seeding and the FWHM of cellular structures, respectively. The blue squares and red triangles show data points for the microfluidic system and the centrifugation method, respectively $(n=3)$. The equations of the fitted lines are FWHM $=14+$ $0.0024 \times($ cell concentration) (plain line) and FWHM $=12+0.0021 \times($ cell concentration $)($ dotted line $)$ using the microfluidic system and centrifugation, respectively.

at the narrowest region were $0.35 \mathrm{~mL} / \mathrm{min}, 4 \mathrm{~mm}$, and $1 \mathrm{~mm}$, respectively. The mechanical force at $100 g$ that was applied to the cells by centrifugation $\left[\rho \times(4 / 3) \times 3.14 \times r^{3} \times 100 \times g\right]$ was calculated using a cell density $(\rho)$ of $1.05 \mathrm{~g} / \mathrm{cm}^{3}$, a spherical cell radius $(r)$ of $10 \mu \mathrm{m}$, and $g$ of $9.8 \mathrm{~N} / \mathrm{Kg} .{ }^{15}$ The mechanical force (0.4 mdyn) that was applied to a cell by centrifugation under these experimental conditions was higher than the force that was applied to a cell by FSS $\left(0.02 \times 3.14 \times r^{2}=0.15 \mu \mathrm{dyn}\right)$. When the flow rate increases and/or the height of the microfluidic channel decreases, the mechanical force applied to the cell in the microfluidic system is smaller than that applied in the centrifugation system. To use this microfluidic system for coating ECMs, the flow rate and channel dimensions should be controlled to prevent damaging the cells at the bottom of the channel. Using this system, the LbL processes are preferable for preventing mechanical damage to the cells by centrifugation. In addition, these processes are less laborious, such as those for several rounds of cell medium removal.

Previous reports of the LbL processes suggested seven LbL coats. $^{3,24}$ We wanted to decrease coating time to save ECM, and multilayers of $\mathrm{C} 2 \mathrm{C} 12$ cells were successfully prepared by coating four times. A recent study has demonstrated that gelatin adhesion on the fibronectin (FN) layer was necessary for thickness enhancement of the FN layer. ${ }^{3}$ A cellular multilayer was not fabricated by only one coat. ${ }^{25}$ Cell adhesion onto cells at a lower layer may be related to the thickness of the BFN layer. Because optimized layer sizes are dependent on cell types, we could not decide the appropriate time for ECM coating processes. Based on our experiments, we can suggest that the ECM coating process is required at least twice, and four times in the LbL process is sufficient for $\mathrm{C} 2 \mathrm{C} 12$ multilayer fabrication. The microfluidic technology for the preparation of LbL ECM films and cellular multilayers can be applied to fabricate model tissues such as blood vessels, which consist of endothelial cells, basal lamina, and fibroblasts. ${ }^{26}$ Furthermore, by coating the bottom surfaces of channels with elastic PDMS, cells in the multilayers can be subjected to compression or stretch stimuli. Through the integration of microfluidic technologies comprising microactuators and/or pumps, it is possible to quantify the responses to either the mechanical or chemical stimulation of 
cellular multilayers. Therefore, it should be feasible to mimic the mechanical forces generated under physiological conditions using the microfluidic LbL system described in this study.

\section{Conclusion}

We developed a microfluidic LbL system comprising three micropumps, two solenoid valves, and a custom-made controller for fabricating cellular multilayers. Our microfluidic system allows for the preparation of cellular bilayered structures in a PDMS-based microfluidic channel. When the ECM-coating method was used to coat cells in the microfluidic channel, the heights of the 3D cellular structures were larger than those of the structure that was fabricated by the centrifugation coating method, and the required labor and mechanical force applied to the cell were reduced. In the future, this microfluidic system can contribute to investigations of cross-talk between different cell layers via the secretion of chemical factors, and to the evaluation of differences in the responses of individual layers to mechanical stimuli.

\section{Acknowledgments}

This study was supported by a grant-in-aid for Scientific Research for Young Scientists (Nos. 22680036 and 26350106 to K. M. and 21700490 to M. M.) from The Ministry of Education, Culture, Sports, Science and Technology (MEXT) of Japan, NEXT Program of the Japan Society for the Promotion of Science (JSPS) (LR026) (M. M.), PRESTO-Japan Science and Technology Agency (M. M.), and Special Coordination Funds for Promoting Sciences and Technology (K. M.) from MEXT of Japan. K. M. thanks Mieko Kodama (Okayama University, Japan) for her assistance with figure preparation and formatting. The authors would like to thank Enago for the English language review.

\section{References}

1. A. Ito, H. Jitsunobu, Y. Kawabe, and M. Kamihira, J. Biosci. Bioeng., 2007, 104, 371.

2. R. F. Mhanna, J. Vörös, and M. Zenobi-Wong, Biomacromolecules, 2011, 12, 609.

3. M. Matsusaki, K. Kadowaki, Y. Nakahara, and M. Akashi, Angew. Chem. Int. Ed., 2007, 46, 4689.
4. K. Kadowaki, M. Matsusaki, and M. Akashi, Biochem. Biophys. Res. Commun., 2010, 402, 153.

5. D. Gao, H. Liu, Y. Jiang, and J. M. Lin, Trends Anal. Chem., 2012, 35, 150.

6. K. Hattori, S. Sugiura, and T. Kanamori, Lab Chip, 2011, 11,212

7. A. Shamloo, N. Ma, M. M. Poo, L. L. Sohnb, and S. C. Heilshorn, Lab Chip, 2008, 8, 1292.

8. J. Wang, J. Heo, and S. Z. Hua, Lab Chip, 2010, 10, 235.

9. J. El-Ali, P. K. Sorger, and K. F. Jensen, Nature, 2006, 442, 403.

10. M. Rossi, R. Lindken, B. P. Hierck, and J. Wester-Weel, Lab Chip, 2009, 9, 1403.

11. W. Tan and T. A. Desai, Biomaterials, 2004, 25, 1355.

12. M. Matsusaki, Bull. Chem. Soc. Jpn., 2012, 85, 401.

13. M. Matsusaki, H. Ajiro, T. Kida, T. Serizawa, and M. Akashi, Adv. Mater., 2012, 24, 454.

14. A. Nishiguchi, H. Yoshida, M. Matsusaki, and M. Akashi, Adv. Mater, 2011, 23, 3506.

15. G. Sauvageau, P. M. Lansdorp, C. J. Eaves, D. E. Hogge, W. H. Dragowska, D. S. Reid, C. Largman, H. J. Lawrence, and R. K. Humphries, Proc. Natl. Acad. Sci., 1994, 91, 12223.

16. E. L. Wilson and G. E. Fransis, J. Exp. Med., 1987, 165, 1609.

17. C. M. Cheng, J. D. Kubicek, and P. R. LeDuc, Appl. Phys. Lett., 2006, 88, 053902.

18. C. M. Cheng, K. Matsuura, I. J. Wang, Y. Kuroda, P. R. LeDuc, and K. Naruse, Lab Chip, 2009, 9, 3306.

19. K. Matsuura, Y. Kuroda, K. Yamashita, and H. Funahashi, J. Reprod. Dev., 2011, 57, 163.

20. K. Watanabe, K. Matsuura, F. Kawata, K. Nagata, J. Ning, and H. Kano, Biomed. Opt. Exp., 2012, 3, 354.

21. S. V. Owen and R. D. Froman, Res. Nursing Health, 1998, $21,557$.

22. S. S. Harris, E. Soteriades, J. A. S. Coolidge, S. Mudgal, and B. Dawson-Hughes, J. Clin. Endo. Metabol., 2000, 85, 4125.

23. N. Sakamoto, N. Saito, X. Han, T. Ohashi, and M. Sato, Biochem. Biophys. Res. Commun., 2010, 395, 264.

24. M. Matsusaki, K. Kadowaki, E. Adachi, T. Sakura, U. Yokoyama, Y. Ishikawa, and M. Akashi, J. Biomater. Sci., 2012, 23, 63.

25. A. Nishiguchi, M. Matsusaki, and M. Akashi, ACS Biomater. Sci., 2015, 1, 816.

26. J. Schmitz, M. Benoit, and K. E. Gottschalk, Biophys. J., 2008, 95, 1448. 